\title{
Monitoring dirofilariasis spread: herding dogs in Armenia
}

\author{
Svetlana Zykova ${ }^{1,{ }^{*}}$, Roman Slobodyanik ${ }^{1}$, Larisa Belova $^{2}$, Andrey Kryazhev $^{3}$ and Aleksey \\ Savinkov ${ }^{4}$ \\ ${ }^{1}$ Perm Military Institute of National Guard Troops, 614112, Perm, Russia \\ ${ }^{2}$ Saint-Petersburg State Academy of Veterinary Medicine, 196084, Saint-Petersburg, Russia \\ ${ }^{3}$ Vologda State Dairy Farming Academy named after N.V. Vereshchagin, 160555, Vologda, Russia \\ ${ }^{4}$ Samara state agrarian University, 446442, Kinel, Samara region, Russia
}

\begin{abstract}
Dirofilariasis is distinguished by the causative agent provoking dirofilariasis: Dirofilaria immitis and Dirofilaria repens. In the territory of Armenia, dirofilariosis is studied little. The article addresses the evaluation of the spread and prevalence of dirofilariasis in Ararat Province of the Republic of Armenia. The research was focused on herding dogs. The research showed that $100 \%$ of cases were caused by D. immitis; the average prevalence among herding dogs in Ararat Province was 16,6\%. Most dogs infected with Dirofilaria were aged 3 to 5 years. Therefore, in the territory of Armenia, there is an active local focus of dirofilariasis in carnivores with the active involvement of herding dogs in its spread
\end{abstract}

\section{Introduction}

The breading of dogs and their use in human activity last for generations and are genetically determined. Centuries of livestock breeding experience make it clear that the breeding of herding dogs and livestock guardian dogs has been playing an important role in the facilitation of hard work of shepherds and enhancement of their efficiency. Natural characteristics of dogs - good sense of smell, good sense of hearing, physical endurance, exceptional attachment to a man, and intelligence - are prerequisite for their herding behaviour and can serve as a basis for their future successful herding training.

Herding dogs are widely used in Armenia as shepherds' helpers to handle herds, protect herds, and look for left behind and lost animals. Herding dogs help shepherds to keep a herd within a particular area, keeping the head together and defending it from wolves. At the same time, herding dogs help to protect fields against being eaten by farm animals.

The purpose of our work is to analyze the spread of dirofilariasis among herding dogs in the Republic of Armenia.

The efficient use of herding dogs is impossible without their correct breeding, management, feeding, and veterinary attendance. As a rule, in a common herd in the rural area of Ararat Province, there are three dogs to pasture and guard the herd. Shepherds like to have relatively not high, light-weight, active, and working dogs.In Armenia, the Caucasian Shepherd Dog is widely spread; it genetically hates wild animals (wolves etc.),

*Corresponding author: zykova.sv@rambler.ru 
fearlessly fights wild animals face-to-face or in team with other dogs; it is suspicious and aggressive towards all strangers [1].

This makes Caucasian Shepherd Dogs indispensible in peasant farms in the Republic of Armenia.

Caucasian Shepherd Dogs' staying with herds in places next to natural water bodies results in the exposure of dogs to the risk of invasive infections transmitted by mosquitoes. In the territory of Armenia such disease is dirofilariasis [2, 3,4].

Dirofilaria immitis and Dirofilaria repens are studied and described. The literature also addresses Dirofilaria honkongensis, which was identified by means of the Knott test and blood smear microscopy followed by Giemsa stain. The nematodes are $280 \mu \mathrm{m}$ to $310 \mu \mathrm{m}$ long and 5 to $8 \mu \mathrm{m}$ wide [5].

V.N. Beklemishev (1970) regarded the parasitic system as a structure consisting of a parasite populations and a host population (or several host populations) ensuring its existence in a biotic community. In biotic communities, population systems of parasitic organisms closely interact with population systems of animals of other types; as a result, a more complex and stable system consisting of several associated animal populations - at least two (a parasite and its host) - appears.

Metabolites of dirofilariasis causative agents exhibit antigenicity.

Scientific researches to study the epizootiology of dirofilariasis and its epidemic projection have been conducted in many countries of the world, and, first of all, they address the definition of possible boundaries of the epizootic process in view of territories, time, and population. In the territory of Armenia, dirofilariasis is studied very little. There are just separate publications dedicated to this problem and addressing some issues of the parasitic disease spread in the 20th century in the territories of the former Soviet Republics. We wanted to define the epizootic process boundaries in the Republic of Armenia. Historically, in the literature, the interrelation between the intensity of the spread of dirofilariasis-infected mosquitoes in places of effluent water accumulation and places most visited by dogs could be seen [6].

The literature outlines climatically similar places having the same problem caused by the spread dirofilariasis $[7,8]$

Dirofilariasis has been also revealed in the countries having common frontiers with the Republic of Armenia. Dirofilariasis caused by D. immitis was registered in Georgia (P.P. Burdzhanadze in 1943). In Azerbaijan, T.P. Khudaverdiyev and Sh.M. Jafarov (1979) studied the spread of dirofilariasis in the Nakhichevan Autonomous Soviet Socialist Republic and found that the maximum infection rates for Dirofilaria (D. repens) were in the lowlands of the republic where vast waterlogged areas were located. In the mountainous area of the republic, dirofilariasis was not revealed. Dogs aged over 5 years were much infected with Dirofilaria[9].

In Iran, according to B. Meshgi, A. Eslami, J. Asharafi (2001) and B. Meshgi, A. Eslami, J.A. Helan (2002), D. immitis was found in blood of $8,4 \%$ of examined dogs in the vicinity of Tabriz. Dirofilariasis infection rate in urban and rural dogs was $4: 1$. The authors found that, in the vicinity of Tabriz, foxes, wolves, and jackals were infected with D. immitis, and they regard that place as one of the most endemic in Iran. The city of Tabriz is the present capital of East Azerbaijan province in Iran; it borders on Syunik Province of the Republic of Armenia along the coasts of the Araxes River [10, 11, 12].

In Turkey, out of 280 of examined dogs $9,8 \%$ were infected with D. immitis $(\mathrm{H}$. Ogeetal.,2003) [13].

The purpose of our research was to define possible territorial boundaries of the epizootic process through the investigation of dirofilariasis infection rate in dogs in Shirak Province, Aragatsotn Province, Armavir Province, Ararat Province, and Syunik Province of 
the Republic of Armenia, as well as to assess the degree of invasiveness of the process (fig. 1).

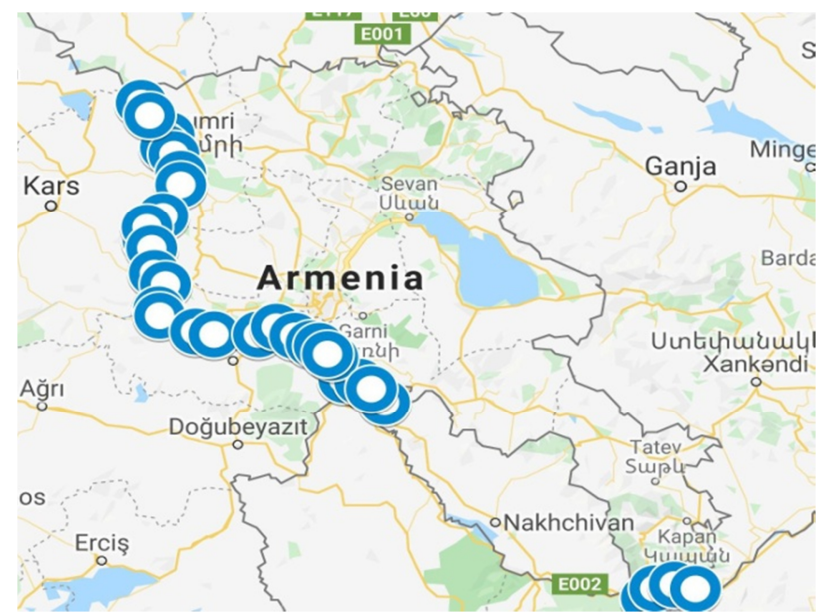

Fig. 1. Regions of studying the epizootic process of dirofilariasis in dogs in farms in Shirak Province, Aragatsotn Province, Armavir Province, Ararat Province, and Syunik Province of the Republic of Armenia.

\section{Materials and methods}

In total we examined 39 herding dogs aged 1 to 5 years (males and females). We examined dogs 2 times a year in the period of active swarming of mosquitoes.

Dogs' venous blood was the material which we analyzed. The laboratory diagnostics included complete blood count conducted with the use of Hematology analyzer Elite 3.

The presence of microfilariae was assessed in direct smears. Adult stage dirofilaria antigen was detected by means of Asan Easy Test Heartworm chromatographic immunoassay kit (manufactured by Asan Farm., Korea) (fig.2).

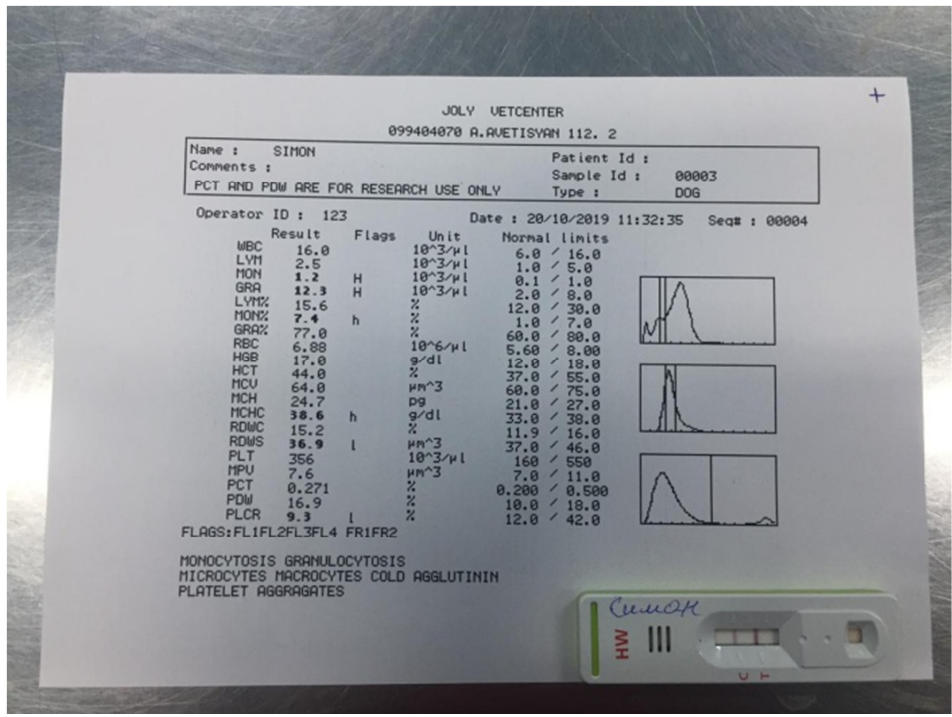

Fig. 2. Complete blood count values and results of the application of Asan Easy Test Heartworm chromatographic immunoassay kit of a dirofilariasis-infected herding dog. 
In addition, the modified Knott method was used; this method allows obtaining higher results at the moment of dirofilariasis diagnosis in dogs compared to blood smear microscopy [14]. According to the method, $10 \mathrm{ml}$ of $2 \%$ solution of acetic acid was added to $1 \mathrm{ml}$ of venous blood. Such solution was mixed well and centrifuged at $1500 \mathrm{rpm}$ for 5 minutes. The supernatant was removed and the precipitated part was mixed with equal volume of methylene blue diluted as 1: 1000and was left for colour staining for 5 minutes. Stained precipitated part was studied microscopically in order to detect fixed microfilariae (fig.3).

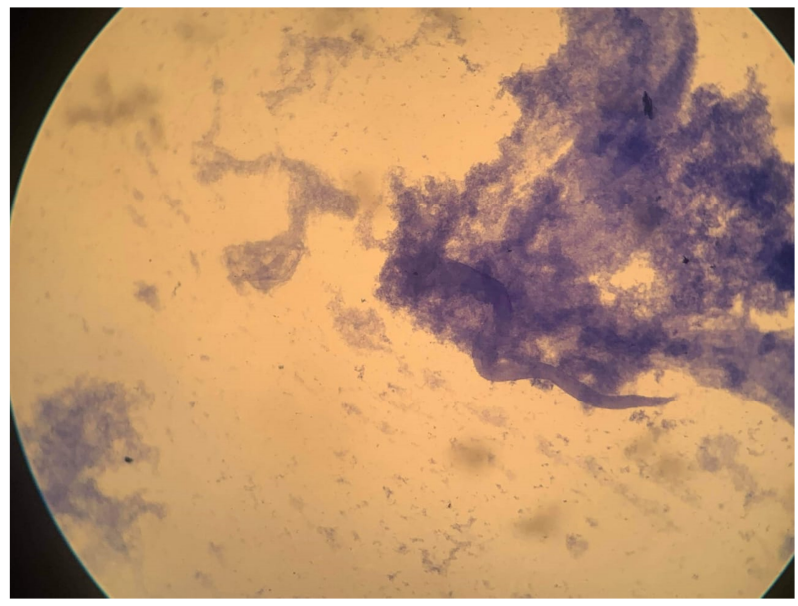

Fig. 3. Microfilaria detected by means of the modified Knott method in blood of a herding dog.

\section{Results}

Dogs' blood was analyzed in three ways: in direct smears, by the Knott test, and with the use of chromatographic immunoassay kit. There was no exact matching between the examination results. The shortcomings of the direct smear method and Knott test include the difficulty to diagnose dirofilariasis is case of low invasion intensity that reduces the accuracy of examinations. The use of chromatographic immunoassay kit gives positive results in respect of Dirofilaria immitis, even when venous blood examination gives false negative results.

Earlier, based on our researches focused on the detection of microfilariae in dogs through the enzyme-linked immunosorbent assay, we found that the causative agent was $D$. immitis, and the average prevalence among dogs in farms in Shirak Province, Aragatsotn Province, Armavir Province, Ararat Province, and Syunik Province was 8,5\%. Among German Shepherd Dogs examined in the same region in the period from 2017 through 2019, dirofilariasis was found in animals aged 3 to 8 years. Dirofilariasis was not found in dogs aged 1 to 3 years. The research included 185 German Shepherd Dogs aged 1 to 8 years (males and females). The prevalence was evaluated in each area of the Republic of Armenia: in Shirak Province the prevalence was 3,6\%; in Aragatsotn Province no cases of dirofilariasis were revealed (the prevalence was 0\%); in Armavir Province the prevalence reached 42,8\%; in Ararat Province the prevalence was 29,6 \%. In Syunik Province no infected dogs were revealed among the selected animals (prevalence was $0 \%$ ). Previously, it was reported that, as of 2017, among German Shepherd Dogs the prevalence was $29,6 \%$ in farms in Ararat Province [4]. In 2019, thanks to veterinary preventive and curative measures, the prevalence dropped to $3,6 \%$. 
In $87,5 \%$ of cases the infection was observed in farms in Armavir Province and Ararat Province, regarded as the most Dirofilaria contaminated zones, where the disease was registered among dogs aged 3 to 8 years. $25 \%$ of cases were revealed in dogs aged 3 to 5 years. $75 \%$ of cases were revealed in dogs aged 5 to 8 years, among which the dogs aged 6 years represented $37,5 \%$, the dogs aged 7 years represented $12,5 \%$, and the dogs aged 8 years represented $25 \%$ [4].

Based on the results of our regular clinical examinations of sick animals and on the assessment of function of their organs and systems in terms of fitness for herding work, we noticed that the first symptoms manifested during physical load, and, in the beginning, were limited to cough. The disease progression led to labored breathing. At the moment of auscultation, crepitation in the lungs and splitting of the second heart sound were detected. The progression of heart failure led to limb swelling and ascites, body weight reduction, and anorexia. Atrial fibrillation and cardiac murmur were observed in the right half of the chest. In case of thromboembolism or massive movement of heartworms, acute symptoms, e.g. "vena cava syndrome", may develop. It manifests as quickly developing dyspnea, paleness of mucous membranes, hemoglobinuria, and murmur over the tricuspid valve and usually leads to death.

The analysis of the dogs showed that, as a rule, shepherds do not keep dogs older than 5 years in their farms. The disease was found in dogs aged 3 to 5 years; externally, the animals did not have any clinical manifestations of the disease. The analysis of hematological parameters and results are shown in the table as an average value and standard deviation based on the Mann-Whitney U test (table 1).

It is seen, that dogs' blood has insignificant monocytosis and leukocytosis which are specific to invasion; reduced hematocrit is an indicator of latent anemia.

All examined dogs aged 1 to 3 years were healthy.

We found that, to a great extent, the spread of the epizootic process in Armenia corresponded to the patterns previously revealed in the Northwest Caucasus in the territory of Russia. It was determined that the location of the epizootic process among carnivores was dependent on mountain relief and climatic conditions. No cases of dirofilariasis were revealed in mountainous areas in the Northwest Caucasus. The highest spread of dirofilariasis was in lowland regions in dogs $(68,8 \%)$, cats $(44,7 \%)$, and jackals $(40,1 \%)$ [15].

Table 1. Hematological values of herding dogs.

\begin{tabular}{|c|c|c|}
\hline Hematologicalvalues & $\begin{array}{c}\text { Physiological } \\
\text { reference values }\end{array}$ & Dogs' analyses results \\
\hline White blood cells (thousand $/ \mathrm{mm}^{3}$ ) & $8.50-10.50$ & $11.25 \pm 1.38$ \\
\hline Lymphocytes & $1.0-5.0$ & $2.35 \pm 0.39$ \\
\hline Monocytes & $0.10-1.00$ & $0.80 \pm 0.09$ \\
\hline Granulocytes & $2.0-8.0$ & $7.60 \pm 1.23$ \\
\hline Lymphocytes.\% & $12.0-30.0$ & $18.95 \pm 3.72$ \\
\hline Monocytes. \% & $1.0-7.0$ & $7.50 \pm 0.36$ \\
\hline Granulocytes. \% & $60.0-80.0$ & $72.80 \pm 3.75$ \\
\hline Red blood cells (million/mm ${ }^{3}$ ) & $5.60-8.00$ & $6.13 \pm 0.40$ \\
\hline Hemoglobin. g \% & $12.00-18.00$ & $13.35 \pm 1.01$ \\
\hline Hematocrit. \% & $37.0-55.0$ & $34.75 \pm 2.50$ \\
\hline Mean corpuscular volume & $60.0-75.0$ & $59.00 \pm 1.56$ \\
\hline $\begin{array}{c}\text { Mean corpuscular hemoglobin } \\
\text { concentration }\end{array}$ & $21.0-27.0$ & $22.80 \pm 0.58$ \\
\hline $\begin{array}{c}\text { Total hemoglobin content in the red } \\
\text { blood cells. mg }\end{array}$ & $33.0-38.0$ & $34.45 \pm 0.17$ \\
\hline Red blood cell distribution width & $11.0-16.0$ & $15.70 \pm 1.00$ \\
\hline
\end{tabular}




\begin{tabular}{|c|c|c|}
\hline Red blood cell distribution.\% & $37.0-46.0$ & $36.70 \pm 1.83$ \\
\hline Platelets & $160-550$ & $470.50 \pm 70.91$ \\
\hline Mean platelet volume & $7.0-11.0$ & $7.40 \pm 0.31$ \\
\hline Thrombocrit & $0.200-0.500$ & $0.310 \pm 0.04$ \\
\hline Platelet distribution width & $10.0-18.0$ & $6.60 \pm 1.75$ \\
\hline Platelet large cell ratio & $12.0-42.0$ & $20.05 \pm 2.77$ \\
\hline
\end{tabular}

Our researches confirm that in the territory of Armenia there is an active local focus of dirofilariasis of carnivores. After the revealing of dirofilariasis in dogs, the deworming was done with the use of moxidectin-based drug, and subsequent follow-upwas arranged [16]. According to international veterinary information, moxidectin exhibits a «dose-response» relationship, and, in some cases, the administration of moxidectin, in the doses recommended by the manufacturer, does not guarantee successful prevention of dirofilariasis. At the same time, increased dose and number of subsequent monthly doses of moxidectin enhances the effectiveness in the treatment of dirofilariasis forms resistant to macrocyclic lactones. Based on this data, $24 \mu \mathrm{g} / \mathrm{kg}$ was chosen as the best dose for further use [17].

\section{Discussion}

Our researches confirm that in the territory of Armenia there is an active local focus of dirofilariasis of carnivores.

There is an opinion that, in the period of clinical manifestations of dirofilariasis, anthelmintics should be administrated with care, as soon as death of a big amount of parasites at all stages of development can provoke the release of significant quantities of somatic antigens in the body of dogs and cats and can cause immediate or remote hypersensitivity reactions. We think that, in the pathogenesis of dirofilariasis, an allergic aspect plays a particular role, and eosinophilia is the symptom thereof. It is found that eosinophilia is caused by the release of antigens having the size which cannot be phagocytized. Eosinophils are able to concentrate around parasites. The scientific literature addresses complex mechanisms of migration of eosinophils to places of parasite location; the concentration of eosinophils requires the participation of immunoglobulins and tissue basophils [7].

Pathomorphological changes in dogs infected with Dirofilaria manifested as an aggregate of general and local pathologic processes. In most animals, nutritional status was below average, their visible mucous membranes were anemic and cyanotic, and animals had eyeball retraction [15].

In all animal species, as a rule, the main location of adult stage $D$. immitisis the right half of the heart and pulmonary arteries. In case of severe infection intensity in dogs, adult stage helminths were found in the aorta, caudal vena cava, blood vessels of the lungs and liver; adult stage D. Immitis were found in the lumen of the bronchi and lungs. In all infected animal species, on the endocardium, there are inflammatory processes: erosive ulcers, necrotizing ulcers, areas of fibrosis/necrosis. Adult stage D. repensin dogs are mostly located in subcutaneous and muscle tissues: in the area of the abdominal wall, rump, shoulder joint, radius bone and ulna of the anterior limbs and on the inner surface of the hind limbs. Microfilariae of the both types, circulating in peripheral blood, cause alterative processes, impairment of hemodynamics in the lungs, kidneys, spleen, digestive tract, and respective regional lymph nodes [15].

The presence of adult stage $D$. immitis in the right half of the heart and in the pulmonary artery represents the etiologic factor for the formation of the cardiac hypertrophy and dilatation in infected animals. 
The literature presents data on morphological changes occurring in the liver. In some areas of the liver, hyperemia of capillaries and protein structure failure were found. Most part of hepatocytes was in the state of adipose degeneration. In some parts there were hepatocytes in the state of granular and hydropic degeneration; hepatocytes with pycnosis and karyolysis were revealed.

In view of a comprehensive approach to the treatment with the use of anthelmintics within the amount of measures necessary to prevent further development of fatty hepatosis, the use of hepatoprotectors exhibiting cytoprotective activity is effective [18-22].

\section{Conclusions}

In the Republic of Armenia, the researchers studied the epizootiology of the causative agent of dirofilariasis in carnivores and determined the spread of dirofilariasis in territories, distribution of its types, and seasonal dynamics of the parasitic disease.

It was revealed that $D$. immitis is the dominating type in helminth associations in herding dogs.

After the revealing of dirofilariasis invasion in dogs, the deworming was done with the use of moxidectin-based drug and subsequent follow-upwas arranged [16]. In order to prevent infection with Dirofilaria, the shoulders of healthy dogs were treated with fipronil solution.

A system of preventive measures should be developed focusing on the reduction of dirofilariasis rate in herding dogs in farms in Ararat Province. The system should include the following measures: 1) regular examinations should be arranged by farm owners for their dogs to check them for dirofilariosis using various methods, such as: direct blood smear microscopy, the Knott test, and the use of chromatographic immunoassay kits; 2) if infected animals are revealed in farms, respective treatment should be arranged; 3) preventive deworming of herding dogs and applying of insecticides and acaricides to herding dogs should be provided; 4) the monitoring of sanitary and epidemiologic conditions of animal management should be arranged, and, in particular, dogs should be kept away from open sources of effluent water (irrigation ditches) which represent the risk factor for the spread of dirofilariosis.

\section{References}

1. J.B. Early, E.R. Arnott, C.M. Wade, P.D. McGreevy, Journal of Veterinary Behavior 14, 1-4 (2016) https://doi.org/10.1016/j.jveb.2016.06.009

2. M. Krstić, S. Gabrielli, M. Ignjatović, S. Savić, G. Cancrini, G. Ranđelović, S. Momčilović, S. Stojnev, S. Otašević, Serbia Molecular and Cellular Probes 31, $37-$ 41 (2017) https://doi.org/10.1016/j.mcp.2016.08.005

3. D. Otranto, P. Deplazes, International Journal for Parasitology: Parasites and Wildlife 9, 370-383 (2019) https://doi.org/10.1016/j.ijppaw.2018.12.011

4. A.L. Kryazhev, R.V. Slobodyanik, International Bulletin of Veterinary Medicine 3, 1621 (2019)

5. S. Gowrishankar, M. Aravind, S. Sastya, B.R. Latha, P. Azhahianambi, S. Vairamuthu, C. Jayanthy, Tamil Nadu. Vet Parasitol. Reg. Stud. Reports 18, 100326 (2019) doi: 10.1016/j.vprsr.2019.100326

6. V.B. Yastreb, Animal Parasitic Diseases: Theory and Practice 12 (2019) https://cyberleninka.ru/article/n/prizhiznennaya-diagnostika-dirofilyarioza 
7. M. Gizzarelli, V. Foglia Manzillo, L. Ciuca, M.E. Morgoglione, N. Houda Ben Fayala, G. Cringoli, G. Oliva, L. Rinaldi, M.P. Maurelli, Stray and Sheep Dogs in a Mediterranean Area. Front Vet Sci. 29(6), 288 (2019) doi: 10.3389/fvets.2019.00288

8. V. Velev, K. Vutova, T. Pelov, I. Tsachev, Helminthologia 56(3), 247-251 (2019) doi:10.2478/helm-2019-0016

9. G. Baneth, S.M. Thamsborg, D. Otranto, J. Guillot, R. Blaga, P. Deplazes, L. SolanoGallego, Journal of Comparative Pathology 155(1), 54-74 (2016) https://doi.org/10.1016/j.jcpa.2015.10.179/

10. C. Genchi, L.H. Kramer, Old World Veterinary Parasitology 21, 108995 (2019) https://doi.org/10.1016/j.vetpar.2019.108995

11. B. Meshg, A. Eslami, J. Asharafi, J. of the Fac. of Vet. Med. 56(3), 115-117 (2001)

12. B. Meshg, A. Eslami, J.A. Helan, J. Fac. Vet. Med. 57(4), 59-63 (2002)

13. H. Oge, A. Doganay, S. Oge, A. Yilidrim, Dtsch. Tierarztl. Wochenschr 110(2), 69-72 (2003)

14. C.C. Evans, J.L. Bradner, M.D. Savadelis, C.T. Nelson, A.R. Moorhead, Vet Parasitol. 276, 108975 (2019) doi: 10.1016/j.vetpar.2019.108975

15. V.M. Kravchenko, Collection of articles based on the materials of the $72^{\text {nd }}$ Academic Workshop of Lecturers (KubGAU, Krasnodar, 2017)

16. T.L. McTier, R.H. Six, A. Pullins, S. Chapin, S.P Mahabir, D.J. Woods, S.J. Maede, Parasit Vectors 11.12(1), 444 (2019) doi: 10.1186/s13071-019-3685-3

17. I.P. Belykh, G.B. Arisova, Russian Journal of Parasitology 13(1), 52-55 (2019) DOI: 10.31016/1998-8435-2019-13-1-52-55

18. A.I. Vengerovsky, A.N. Melentyeva, V.N. Burkova, J. of Chem. and Pharm. B.44.3, 29-31 (2010)

19. Y. Yue, Q. Lin, M. Irfan, Q. Chen, X. Zhao, Bioresour Technol 220, 378-383 (2016) DOI: 10.1016/j.biortech.2016.08.028

20. M.P. Semenenko, N.N. Zabachta, N.N. Sokolov, E.V. Kuzminova, J. of Pharm. Sci. and Res. 10(1), 146-147 (2018)

21. M.P. Semenenko, E.V. Kuzminova, F.D. Onishchuk, E.V. Tyapkina, Veterinary Med. 4, 42 (2016)

22. G. Wardani, N. Farida, R. Andayani, M. Kuntoro, S.A. Sudjarwo, Pharmacognosy Res. 9(3), 282-286 (2017) DOI: 10.4103/pr.pr_69_16 